\title{
A review of the therapeutic potential, prospects, and challenges of CRISPR/Cas9 genome editing in the treatment of Sickle Cell Disease (SCD)
}

\author{
Arundhati Chaudhary ${ }^{*}$, Barsha Kumari ${ }^{* *}$, Meghna Choudhury ${ }^{* * *}$, Shubham Singh ${ }^{* * * *}$, Dr. Ramneet Kaur ${ }^{* * * * *}$ \\ * Department of Biomedical Sciences, Acharya Narendra Dev College, University of Delhi, Delhi \\ ** Department of pharmaceutical science, Parul University, Vadodara, Gujarat \\ *** Dr. Vithalrao Vikhe Patil Foundation's Medical College, Ahmednagar, Maharashtra (MUHS) \\ ***** Department of Botany, Centre of Advanced Study, Banaras Hindu University (BHU), Varanasi Uttar Pradesh \\ ${ }^{* * * * *}$ Assistant Professor, Department of Basic and Applied Sciences, RIMT University \\ DOI: 10.29322/IJSRP.11.01.2021.p10983 \\ http://dx.doi.org/10.29322/IJSRP.11.01.2021.p10983
}

\begin{abstract}
As sickle cell disease is a well-known genetic disorder of point mutation, it is considered a leading candidate for geneediting therapies. Studies published in 2016 described a successful proof-of-concept in treating sickle cell disease in mice using the CRISPR-Cas9 gene-editing tool. CRISPR -Cas9 is a programmable RNA-guided DNA endonuclease, which has been gaining significant attention over the last decade due to its ability to treat genetic disorders such as sickle cell disease. Guided by RNA strand, the Cas9 nuclease- originally isolated from bacteria can be programmed to cut a target DNA sequence and modified by inserting, deleting, or replacing it with the normal copy of the genetic sequence. Though successfully demonstrated in mice species, this genome editing tool is still in a very nascent stage concerning utilization in the human population. Through this review paper, we analyze the scope, possibilities of CRISPR-Cas9 as a potential therapeutic tool in the management of sickle cell disease.
\end{abstract}

Index Terms- Sickle Cell Disease, CRISPR-Cas9, programmable endonuclease, gene therapy

\section{INTRODUCTION}

$\mathrm{S}$ ickle cell disease or as an abbreviation (SCD) is an Inherited disease caused by point mutations in the beta-globin gene that helps make (Hb). Normally, hemoglobin in RBCs takes up oxygen in the lungs and carries it through the arteries to all the cells in the tissues of the body. RBCs that contain normal hemoglobin are disc-shaped and flexible so that they can move easily through large and small blood vessels to deliver oxygen. However, in the Sickle hemoglobin $(\mathrm{HbS})$ unlike normal hemoglobin $(\mathrm{Hb})$ the mutations in the gene lead to problems when oxygen levels in the blood are less, which occurs once the hemoglobin has delivered oxygen to the cells in the body's tissues ${ }^{[1]}$. With less oxygen, the abnormal hemoglobin $\mathrm{S}$ gene can cause rigid, non-liquid protein strands to form within the RBCs which eventually lead to the change in the shape of the cell making it sickle-shaped which are not flexible and adhere to vessel walls, causing blockage to the flow of the blood. When this happens, an adequate amount of oxygen doesn't reach the destined tissues. This lack of supply makes up the etiology for severe pain known as 'crises' which is often seen in patients with SCD. Due to the apparent change in morphology of the RBCs, they tend to burst up easily followed by a decline in longevity by 10-20days from regular 90-120 days. Furthermore, the number of RBCs is lower than usual which leads to the condition in the patients called Anemia with less energy and feeling weakness and lethargy in patients. Genetically speaking, in homozygous conditions RBCs of SCD patients produce $\mathrm{HbS}$ and lack HbA because they inherit two alleles of the HbS gene. On the contrary, people with the heterozygous show (SCT) Sickle Cell Trait with the single allele of both the HbS and HBB gene and usually do not experience symptoms of SCD due to the coexistence of normal $\mathrm{HbA}$ and $\mathrm{HbS}$ in RBCs. Currently, an allogeneic bone marrow transplant is the only potential approach to cure SCD However, in clinical practice, locating a suitable donor is difficult and the allogeneic marrow transplant procedure has serious risks, including patient death considering this, the therapeutic rationale to treat SCD patients can be founded on the conversion of SCD to SCT genotype via genome editing of $\mathrm{HbS} / \mathrm{HbS}$ to $\mathrm{HBB} / \mathrm{HbS}$. Clustered regularly interspaced short palindromic repeats (CRISPR) and CRISPR-associated (Cas) genes were initially discovered in Escherichia coli In 2007, Barrangou et al. demonstrated that integrating a genome fragment of an infectious virus into its CRISPR locus conferred Streptococcus thermophilus resistance against a bacteriophage. In 2012, Jinek et al. demonstrated the capacity of the CRISPR/Cas9 system to perform RNA-programmed genome editing. This approach for genome editing has been studied in a variety of organisms like bacteria, yeast, Drosophila, Zebrafish, plants, monkeys, pigs $^{[1]}$. These findings provide a solid foundation to treat SCD by genome editing of patient HSPCs using the CRISPR/Cas9 approach.

\section{HISTORY}

"Peculiar elongated and sickle-shaped" is how sickle cells were first described by Ernest. While examining the first-ever patient Noel from Black Family in Grenada suffering from anemia who later on died of pneumonia. It was the first known case in the States, however, the ancestry of the disease goes back to literature 
that reported this condition in the 1870 s where reports of this condition were traced back to 1670 in one Ghanaian family. Originally 1910 is the embarked date of the discovery of the disorder we call 'Sickle Cell Disorder' or (SCD). Although it has been scripted in many tribal languages of Africa, credit for being entitled as molecular disease or disorder goes to US cardiologist Dr. James B. Herrick and Dr. Ernst. As approached by a patient with a complaint of pain episodes and symptoms of anemia, being a Cardiologist he was least interested and handed it to Ernst who when studied the sample of blood under a microscope found RBCs as 'being sickle-shaped' which made Dr. Herrick was intrigued about the unknown and finding and he immediately wrote a paper in one of Medical Journals where he phrased the cells as 'sickleshaped cells'. As the unraveling of the thick layers of disease began, In 1927, Hahn and Gillespie RBCs from persons with the disease could be made sickle by removing the O2. Since RBC is the oxygen carrier in the body, it was a profound finding of the time. However, the problem begins when it was analyzed that the relative of the patients with this trait was not indicting disease condition. So, this confusion persisted until the late 1940s. Interestingly, a Revolutionary finding came to light when two independent papers each in Africa by Dr. Beet and in the US by Dr. Neel was published. Although, the paper which was published in US cough many audiences, the finding of articles showed conclusively that SCD was inherited and the people with sickle trait was carrier (heterozygous/AS) for the gene whereas the people with the disease were (Homozygous/SS) for a gene. Later, In 1951, the Nobel Laureate Dr. Linus Pauling and Dr. Harvey
Itano discovered that the red, oxygen-carrying protein called "hemoglobin" had a different chemical structure in persons with SCD. However, the most significant finding of the substitution of the amino acid residue was worked out by Dr.Vernon Ingram in 1956. The origin of the mutation that led to the sickle-cell gene derives from at least four independent mutational events, three in Africa and a fourth in either Saudi Arabia or central India. These independent events occurred between 3,000 and 6,000 generations ago, approximately 70-150,000 years. A significant figure in Sickle Cell history is Dr. Charles F. Whitten. He was a trailblazer in medical education and sickle cell disease awareness. During the early 1970s, he realized that services for people with sickle cell disease and their families were sorely lacking. Out of his pioneering efforts to advocate for the needs of people with sickle cell disease, the Sickle Cell Disease Association of America (SCDAA) was born. Despite these and many other advances that have greatly improved and helped lengthen the lives of those with sickle cell, there is still no universal cure. Genetic counseling became an important tool for informing people about the risks of having a child with sickle cell disease. In the United States, it is estimated that over 80,000 people have sickle cell disease. Over 1,000 babies are born with the disease each year in America. Today, 100 years later, physicians and scientists continue to move forward in a new understanding of the disease and new ways to treat it. The goal of a total cure has not been reached but great progress has been made. Perhaps within the lifetime of some of us, that goal will be reached.

\section{PATHOPHYSIOLOGY}

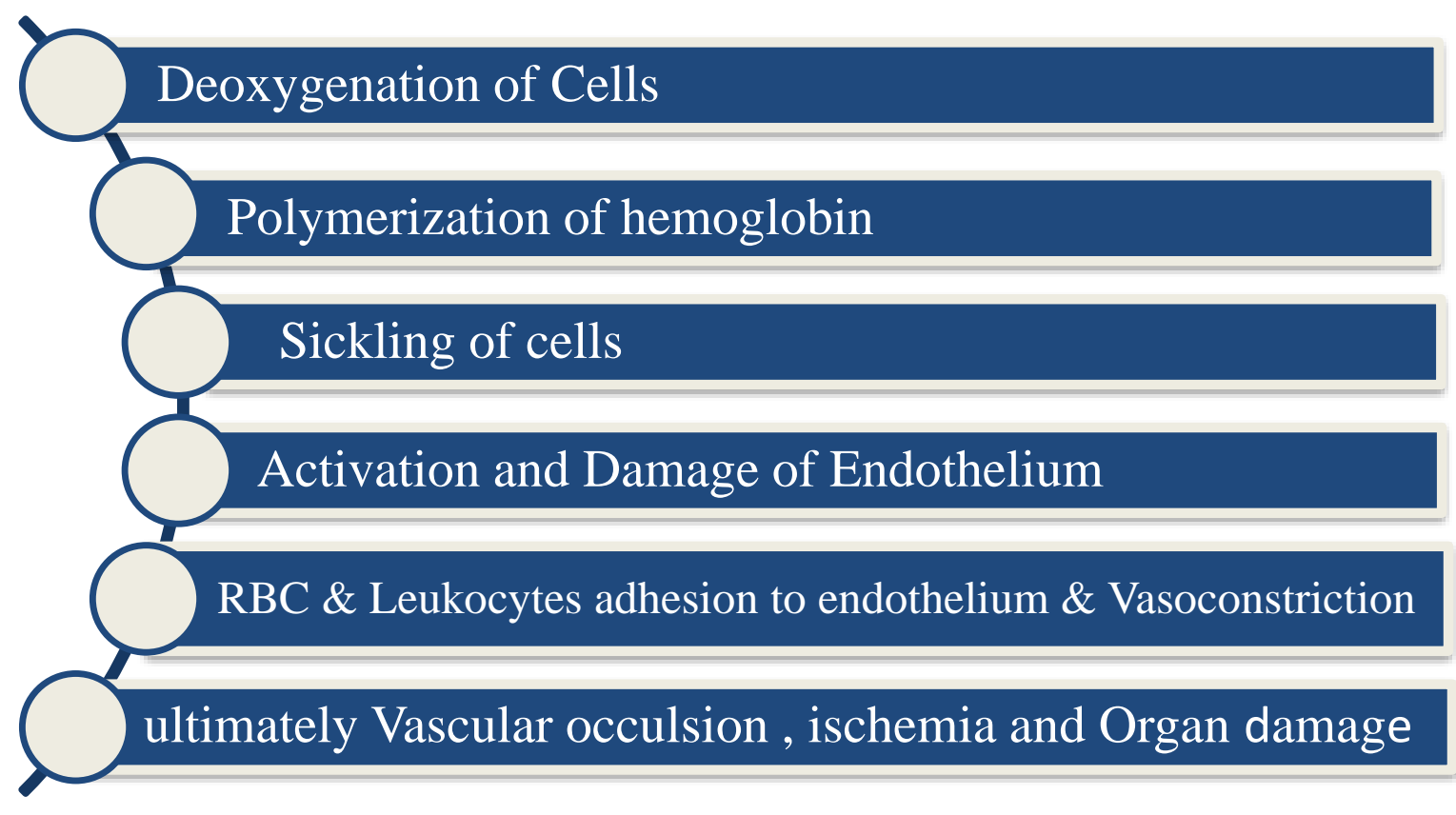




\section{SICKLE CELL DISEASE AT MICROSCOPIC LEVEL}

Sickle Cell Disease is an inherited autosomal recessive disorder, resulting from point mutation at the beta-globin gene in the short arm of Chromosome 11. To understand how sickle cell disease is passed on, it is imperative to understand the genetic basis of the disease.

The normal hemoglobin molecule consists of 4 subunits- 2 alpha and 2 beta subunits. Each subunit is composed of a polypeptide chain - globin, and a prosthetic group- heme, an ironcontaining pigment that binds to oxygen and gives the molecule its oxygen transporting ability. Each globin chain is made of 8 segments, from $\mathrm{A}$ to $\mathrm{H}$, and heme is inserted between $\mathrm{E}$ and $\mathrm{F}$. The synthesis of hemoglobin is directed by the beta-globin genes which are organized into two clusters - the alpha-like genes and the beta like genes. The alpha-like genes are found on the short arm of chromosome 16 (16p) which contains 3 functional genesalpha1, alpha2, delta2, 3 pseudogenes, and 1 gene of undetermined function. The alpha1 and alphal and 2 have close nucleotide sequences and an identical coding sequence. The beta-like genes are located on the short arm of chromosome 11 (11p). It contains 5 functional genes and 1 pseudogene.
The point mutation observed in SCD replaces " $A$ "with " $T$ " at codon 6 of the beta-globin chain. Consequently, it alters the amino acid sequence, replacing glutamic acid with valine. The valine makes the hemoglobin molecules stick together forming long fibers that distort the shape of the RBCs and this brings on the sickling at low oxygen tension. Consequently the product- $\mathrm{HbS}$ is an insoluble protein as opposed to the $\mathrm{HbA}$ which is soluble. The solubility allowed the RBC molecule to carry out its oxygentransporting function. The insoluble $\mathrm{HbS}$ causes the sickled $\mathrm{Hb}$ molecules to aggregate as rod-shaped polymers or fibers under low oxygen tension, and hampers the function of erythrocytes, causing bouts of ischemia and pain in the subject.

The inheritance follows an autosomal recessive pattern. It gives rise to 2 clinical entity-

1. Sickle Cell Trait- the presence of 1 copy of HbS

2. Sickle Cell Anaemia- the presence of 2 copies of $\mathrm{HbS}$, one from each parent.

- HGNC Approved symbol; $H B B$

- Cytogenetic location : $11 p 15.4$

- Genomic coordinates (GRCh38): 11:5,225,463$\underline{5,227,070}$

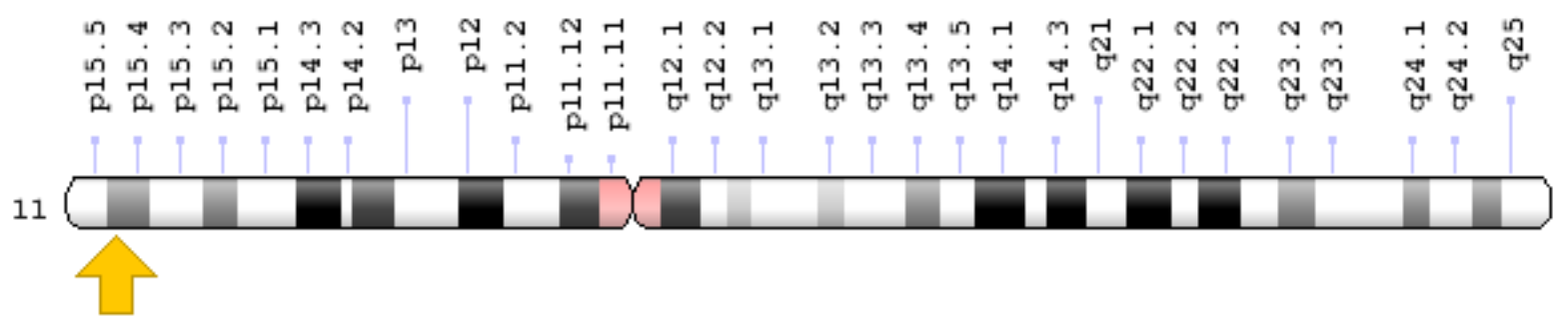




\section{GENE EDITING WITH CRISPR}

CRISPR-Cas9 gene editing is helping to tackle sickle-cell disease in two ways.

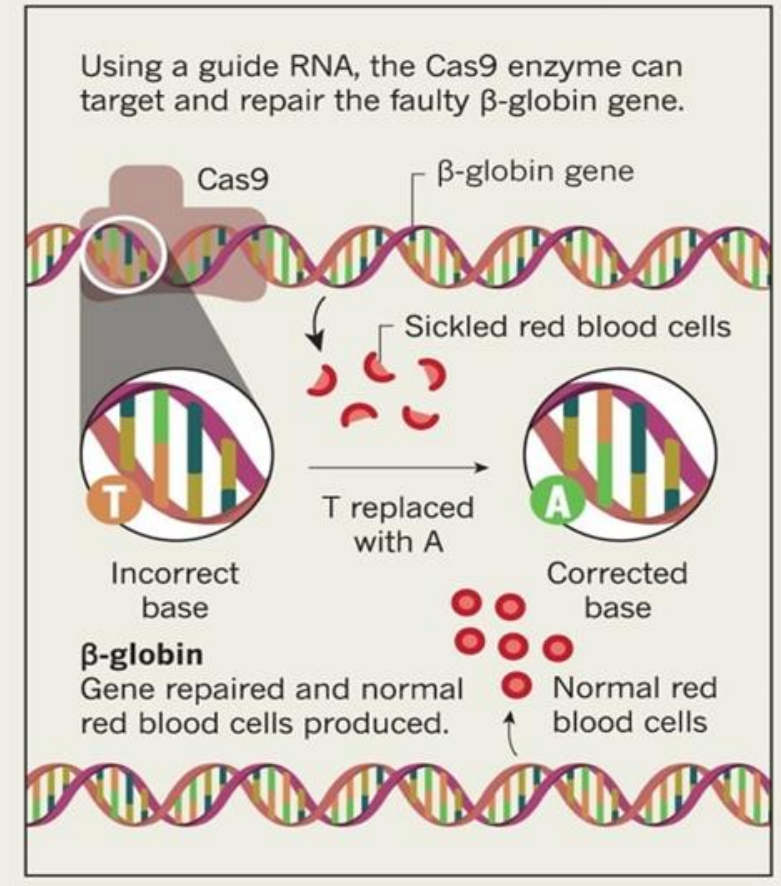

Every cell in our body contains a copy of our genome, with 20000 genes and 3 billion letters of the DNA.DNA consists of 2 twisted strands of double helix held together with a simple pairing rule. Pairs with $\mathrm{T}$ and $\mathrm{G}$ pairs with $\mathrm{C}$. Our genes shape who we are as individuals and as a species. Genes also have profound effects on health, and due to advances in DNA sequencing, researchers have found thousands of genes that affect our risk of disease. To understand how genes work, researchers need ways to control them. Changing genes is not an easy feat, however, due to recent advances, we can now think of this possibility.

The new wonder molecule, in the world of biotechnology that has redefined how we can approach pathological conditions is the CRISPR Cas9 genome editing tool. But what exactly is it? It stands for Clustered Regularly Interspaced Short Palindromic $\underline{\text { Repeats - CRISPR Associated Genes. }}$

It is understood that bacteria have a natural defense mechanism against bacteriophages. The bacteriophage can infect the bacteria by incorporating its genetic material into the bacterial genome during the first bout of infection. However the second time around, the bacteria recognize the phage genome and produce - g-RNA OR guide-RNA.Cas9 is a nuclease- a type of enzyme that can cut DNA. The CRISPR array, which is always found in association with the Cas9, contains spacers very similar to the phage DNA. The spacers came to be known as the short palindromic repeats- and we soon understood to be the bacteria's defense mechanism against the phage. The spacers are the history of the bacteria's infections, which are formed after every time the bacterial cell encounters an infection. The CRISPR sequences are transcribed containing short CRISPR RNA molecules and the spacer molecules being palindromic repeats, are complementary

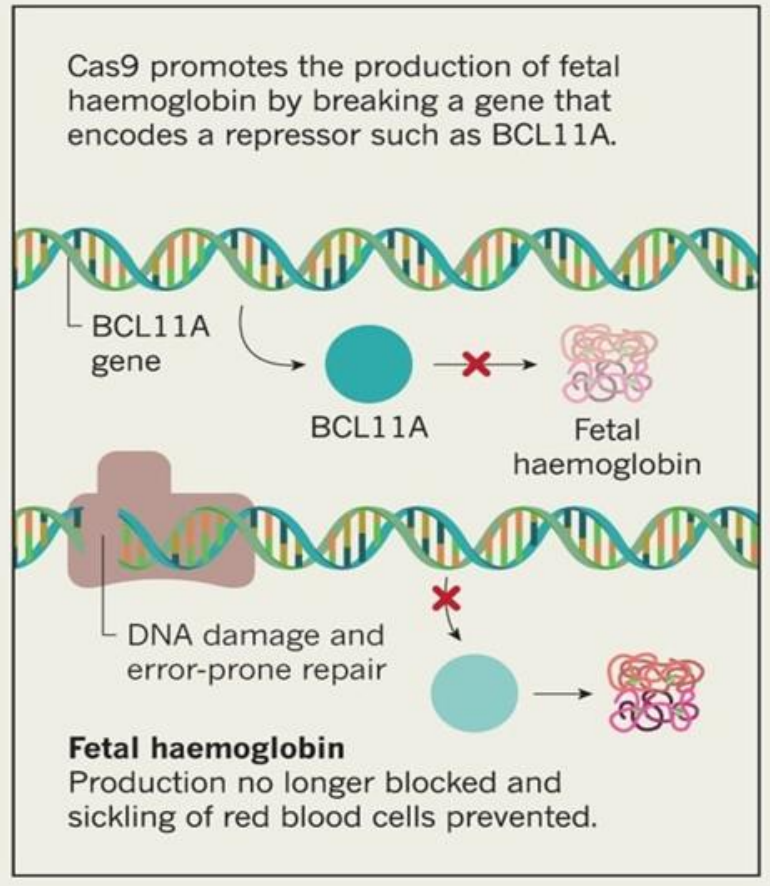

in nature, and its transcription finally leads to the formation of hairpin structure- the guide-RNA. The CRISPR-Cas9 system is in essence the immunological system of the bacteria.

When the matching sequence, known as the guide RNA, finds its target in the phage genome, the cas9 cuts the phage DNA, disabling it. The g-RNA produces a constant sequence and a variable sequence. The constant sequence binds to the Cas9 system and the variable sequence binds to the phage DNA. The Cas9 is a nuclease system with the potential to break the phage DNA. The combined action of the g-RNA and Cas9 brings about a break in the phage DNA.

Once inside the nucleus, the resulting complex will lock onto a short complex known as PAM. The cas9 will unzip the DNA and match it to its target RNA. If the match is complete the cas9 will use 2 tiny molecular scissors to cut the DNA. When this happens, the cell tries to repair the cut, but the repair process is error-prone leading to a mutation that can disable the gene, allowing researchers to understand its function. These mutations are random but sometimes researchers need to be more precise, for example by replacing a mutant gene with a healthy copy. This can be done by adding another piece of DNA that carries the desired sequence. Once the CRISPR system has cut, this DNA template can pair up with the cut ends, recombining and replacing the original sequence with the new version. All this can be done in cultured cells including stem cells that can give rise to many different cell types. It can also be done in fertilized eggs allowing transgenic animals with targeted mutations. Unlike previous methods, CRISPR can be used to target multiple genes simultaneously, facilitating the therapy of not just diseases with point mutations but also polygenic mutations. These methods are 
being improved rapidly and are being used in basic research - drug development, agriculture, and for treating disorders of genetic origin in humans.

Over the past few years, researchers studying the system realized that it could be engineered to cut not just viral DNA but any DNA sequence at the precisely chosen location by changing the guide RNA to match the target. This can be done, not just in a test tube but also within the nucleus of a living cell.

The manifestation of the Therapeutic Potential of CRISPR-Cas9 for SCD

For one sickle cell patient, Victoria Gray who has been suffering from this crippling disease her entire life, the possibility to do away with it, permanently, irreversibly, and with the prospect of protecting her future generations from it, was gifted to her by this new technology of CRISPR Cas9. Much of her life has been punctuated by trips to the emergency rooms due to pain, with life and career ambitions put on hold or interrupted. It has progressed to hamper her heart functioning, and strokes are always a constant threat. It is the most common inherited blood disorder. The alleviation of symptoms and the burden of disease by marrow transplant remains the only option to this day for many, while for a whole lot of others, there is a bleak hope of the future due to the rapid advancement of the disease.

With rumination over getting a bone marrow transplant for the same, Victoria's doctors instead suggested something different to try and treat her disease. She jumped at the chance and went on to become the first patient to be listed and treated with the CRISPR-Cas9 genome editing tool. Come August 2020, a year later from her commencement of treatment, this modality appears to be working better than any other. The billions of genetically modified cells infused in her body appear to be alleviating virtually all the complications of her SCD. Let us understand how this therapeutic miracle was brought about.

The CTX001- While Victoria Gray's SCD is a genetic disorder, the mode of genetic intervention did not involve deleting and adding a healthy copy of the beta-globin gene in her DNA. Instead, the CTX001 is employed in its therapy. The CTX001 boosts the production of fetal hemoglobin HbF- the type of hemoglobin that is present at birth but stops being produced in adulthood. The principle of CTX001 is that the production of $\mathrm{HbF}$ can compensate for the defective $\mathrm{Hb}$ produced.

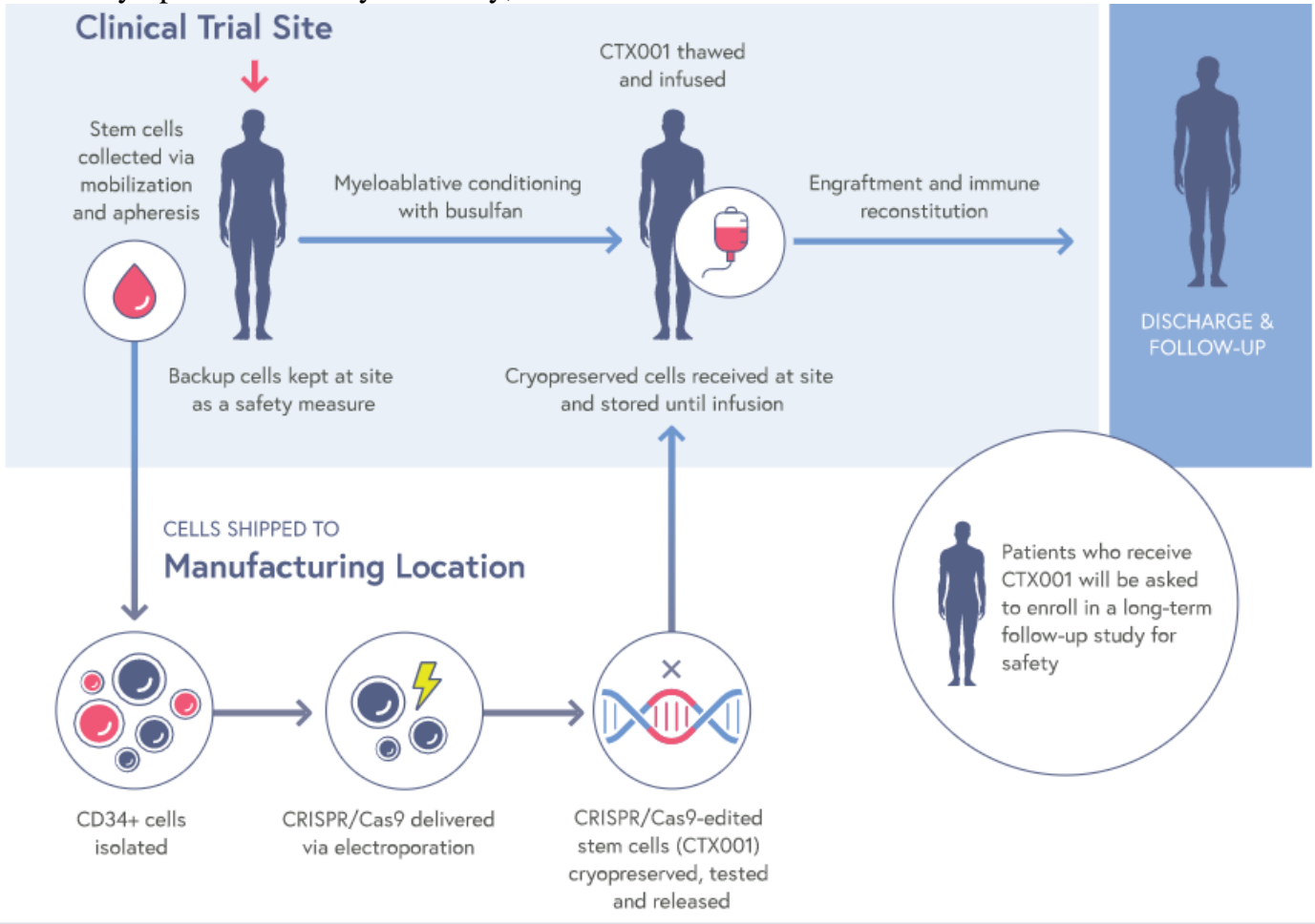

http://www.crisprtx.com/programs/hemoglo

The steps involved are-

1) Harvesting stem cells from a patient's bone marrow.

2) Delivery of CRISPR-Cas9 component to the cells.

3) The action of CRISPR Cas9- deletion in gene Bcl-11a( it encodes a transcription factor which normally represses fetal hemoglobin synthesis)

4) The consequence of the CRISPR-Cas9 action- Break in the gene Bcl-11A.

5) Resumed action of the cells to produce the HbF protein.
6) Production of billions of such modified and corrected stem cells.

7) Reinfusion of these stem cells. When a majority of the stem cells transplant in the body and produce the protein, it compensates for the production of $\mathrm{HbS}$ and effectively controls the disease.

Prognosis and outcome- The predicted levels of $\mathrm{HbF}$ in the patient was $20 \%$, at the initiation of the treatment in 2019. In August 2020, the HbF levels in the patient far exceeded the expectations of the doctors, with the HbF levels being $46 \%$ of the total hemoglobin. Additionally, $81 \%$ of the bone marrow in her 
body contains the genetic modification through stem cells which are needed to produce the fetal hemoglobin protein. It indicates that the edited and modified stem cells have continued to survive in her body for an extended period, much greater than what was expected.

Quality of life post-therapy- From repeated trips to the emergency wards and multiple transfusions needed to survive and continue with life, to zero hospitalizations and alleviation of symptoms and complications post CRISPR-Cas9 gene therapy, Victoria Gray's life took a full turn for the good. While she was the first SCD patient to receive this experimental treatment, she is not the only one. In 2019 patients with beta-thalassemia have also been receiving CTZ001 treatment for pumping up fetal hemoglobin level through stem cell modification.

A critique- Though gene therapy can treat many but not all of these disease complications, it still hasn't been deemed as a cure for the same. The CTX001 does not come without downsides, like any other treatment modality. The patient is deemed to undergo a session of chemotherapy to eliminate their bone marrow and make space for the new cells. Akin to chemotherapy for cancer patients, and transplant patients, the side effects remain the same - fatigue, nausea, mouth sores, bleeding, prone to infections due to immunosuppression, hair loss, loss of appetite. Hence, though the gene therapy effectively curbs the disease, it still comes with adverse effects that the patient would inevitably face. The second most important factor which acts as a drawback is the cost of the therapy. With millions suffering from SCD, gene therapy is a distant dream to be manifested into reality simply owing to the financial constraints. Bone marrow transplant which has remained the mainstay of the treatment, also remains unattainable for a large number of such patients, consequently contributing to the mortality rate of the disease. Although the results are extraordinarily promising, the bigger questions which still loom are-

1. For how long will the treatment last?

2. What complications and adverse reactions are we yet to expect?

3. What are the contraindications of the therapy?

4. Is it safe in the long term?

5. Will it help the patients live longer?

Future \& Challenges

Consequently, many researchers have sought improvements to CRISPR with the gene-editing method expected to continue development well into the future.

CRISPR systems to target lots of genes, researchers at ETH Zurich in Switzerland swapped the Cas9 enzyme for Cas12a. Using this plasmid allowed the researchers to simultaneously edit genes in 25 target sites. The team predicts that dozens or even hundreds more sites could be modified using this method.

The team says that this research contributes to an enhancement of CRISPR technologies as it provides a potential alternative for CRISPR-Cas9 when there are complications such as immune responses to Cas proteins.

There are many benefits of CRISPR/Cas9 systems that can be utilized using in vitro laboratory engineering. However, the full realization of the potential of CRISPR/Cas9 approaches will require addressing many challenges. Within the system itself, offtarget cutting remains a problem. Cas9 nickases and mutants that

This publication is licensed under Creative Commons Attribution CC BY.

http://dx.doi.org/10.29322/IJSRP.11.01.2021.p10983 reduce non-specific DNA binding have been engineered specifically to ameliorate this problem, though they are an imperfect solution. Extensive efforts have been made in understanding sgRNA binding and mismatch tolerance, leading to the development of several predictive software sgRNA design tools; however, our understanding of off-target effects remains poor. This is a vital area for continued study of CRISPR/Cas9 is to realize its promise.

Regarding gene cargo delivery systems, this remains the greatest obstacle for CRISPR/Cas9 use, and an all-purpose delivery method has yet to emerge. Instead, multiple methods are seen for delivering CRISPR to cells. Every method has both advantages and disadvantages, and some can be quite specific or ill-suited to certain types of delivery (e.g. delivery to cells in a flask vs. delivery to a living organism). Further, the best geneediting results with minimal off-target effects are generally obtained from delivery of the ribonucleoprotein, as opposed to plasmid DNA or mRNA. Currently, there are more options for the delivery of small-molecule cargo than for the relatively large protein-nucleic acid complex. The development of new delivery approaches that enable effective RNP delivery will make a meaningful impact on the field. Still another barrier for delivery systems is ensuring that the chosen system is both safe and specific. Safety in living organisms will always be a concern, and a delivery vehicle that can target the desired cells with highspecificity will also limit off-target effects and improve safety. Additionally, it is vitally important that, especially in the case of nanoparticle carriers, long-term studies on the safety of the component pieces are done. There is currently limited information available on where various components of nanoparticle delivery systems end up in the body, how long they stay there, and whether there is any long-term toxicity associated with any component.

As evident through the many examples presented in the 'Delivery Methods' portion of this review, the therapeutic potential of CRISPR/Cas9 is great. Already, much has been published on the altering of cell line genotypes and phenotypes using this gene-editing system. Work has even moved into animal models, and therapeutic effects are broad-ranging, including inhibition of viral infection, a reversal of debilitating conditions such as muscular dystrophy, and elimination of tumors in cancer models. Taken together, it is easy to see the reason for so much excitement in the CRISPR field. As the technology evolves and CRISPR becomes even more mechanistically precise and can be delivered with ever-increasing precision, its therapeutic potential will continue to rise.

\section{CONCLUSION}

CRISPR/Cas9 system will undoubtedly revolutionize the study and treatment of both immunological and allergic diseases. Concerned authorities should formulate the Authorize such laws and regulations that permit the safe and ethical use of this emerging technology for basic research and clinical purposes.

With the advancement in research in this direction, researchers are optimistic to find the answers to these questions in the time to come. The extraordinary feat to treat diseases at their root as opposed to symptomatic relief will bring new surges of treatment modalities in modern medicine. The results are promising, and there is still a lot to be done. The scientists are still 
dealing with the tip of the iceberg which CRISPR-Cas9 is. At this point, with speculations abound, we still cannot pinpoint how it would shape the future of our species and those around us, but the impact and repercussions from what we've known from our history are, that is would be huge. Science has progressed a long way in understanding genetics. Genes, through their expression in the form of proteins, remain the metaphorical "gun", with the environment in the role of the "trigger". Examples of identical twins with marked differences in behavior and lifestyle, and plant modifications without genetic alterations- are a testament to the fact that genes are not the be all end all of our existence, and neither are we slaves to our genome. Epigenetics would play a significant role in modifying disease processes and progressions in the future. Until then, we can wonder, and engage in discourses of the potential and prospects of miraculous advancements in science during our lifetime.

\section{ACKNOWLEDGMENTS}

We express our gratitude to everyone who supported us and to Dr. Ramneet Singh for the keen interest she took in us. She explained out to us solutions to the problems that had a straining effect. Without her help, it was a matter of acute impossibility to propel in this endeavor. It is a matter of profound privilege, and pleasure to pay our sincere and heartfelt thanks to her. We are also very grateful to the almighty and other pioneers whose astounding work has allowed us to share our perspective on this subject.

\section{REFERENCES}

[1] Wen, Jianguo, et al. "Cellular function reinstitution of offspring red blood cells cloned from the sickle cell disease patient blood post CRISPR genome editing." Journal of hematology \& oncology 10.1 (2017): 1-11.

[2] Demirci, Selami, et al. "CRISPR/Cas9 for sickle cell disease: applications, future possibilities, and challenges." Cell Biology and Translational Medicine, Volume 5. Springer, Cham, 2019. 37-

52
[3] Lino, Christopher A., et al. "Delivering CRISPR: a review of the challenges and approaches." Drug delivery 25.1 (2018): 1234-1257.

[4] Dever, Daniel P., et al. "CRISPR/Cas9 $\beta$-globin gene targeting in human hematopoietic stem cells." Nature 539.7629 (2016): 384-389.

[5] Doudna, Jennifer A., and Emmanuelle Charpentier. "The new frontier of genome engineering with CRISPR-Cas9." Science 346.6213 (2014).

[6] Jiang, Fuguo, and Jennifer A. Doudna. "CRISPR-Cas9 structures and mechanisms." Annual review of biophysics 46 (2017): 505-529.

[7] Sürün, Duran, Harald von Melchior, and Frank Schnütgen. "CRISPR/Cas9 genome engineering in hematopoietic cells." Drug Discovery Today: Technologies 28 (2018): 33-39.

[8] Park, So Hyun, et al. "Therapeutic CRISPR/Cas9 genome editing for treating sickle cell disease." (2016): 4703-4703.

[9] Zhang, Feng, Yan Wen, and Xiong Guo. "CRISPR/Cas9 for genome editing: progress, implications, and challenges." Human molecular genetics 23.R1 (2014): R40-R46.

[10] Brodsky, Robert A., and Michael R. DeBaun. "Are genetic approaches still needed to cure sickle cell disease?." The Journal of Clinical Investigation 130.1 (2019).

[11] Orkin, Stuart H., and Daniel E. Bauer. "Emerging genetic therapy for sickle cell disease." Annual review of medicine 70 (2019): 257-271.

\section{AUTHORS}

First Author - Arundhati Chaudhary, Department of Biomedical Sciences , Acharya Narendra Dev College, University of Delhi, Delhi, Email ID - arundhatichaudhary1010099@gmail.com

Second Author - Barsha Kumari, Department of pharmaceutical science, Parul University, Vadodara, Gujarat, Email ID- barshakumari818@gmail.com

Third Author - Meghna Choudhury, MBBS, Dr. Vithalrao Vikhe Patil Foundation's Medical College, Ahmednagar, Maharashtra (MUHS), Email ID -

Choudhury.meghna14@gmail.com

Fourth Author - Shubham Singh, Department of Botany Centre of Advanced Study, Banaras Hindu University (BHU)

Varanasi Uttar Pradesh, Email ID - 1697shubham@gmail.com

Fifth Author - Dr. Ramneet Kaur, Assistant Professor, Department of Basic and Applied Sciences, RIMT University Email ID - ramneetkaur@rimt.ac.in 